University of Windsor

Scholarship at UWindsor

9-23-2015

\title{
A Self-Assembled, Low-Cost, Microstructured Layer for Extremely Stretchable Gold Films
}

\author{
Tricia Carmichael \\ University of Windsor \\ Heather L. Filiatrault \\ University of Windsor \\ R. Stephen Carmichael \\ University of Windsor \\ Rachel A. Boutette \\ University of Windsor
}

Follow this and additional works at: https://scholar.uwindsor.ca/chemistrybiochemistrypub

Part of the Materials Chemistry Commons

\section{Recommended Citation}

Carmichael, Tricia; Filiatrault, Heather L.; Carmichael, R. Stephen; and Boutette, Rachel A.. (2015). A SelfAssembled, Low-Cost, Microstructured Layer for Extremely Stretchable Gold Films. ACS Applied Materials and Interfaces, 7 (37), 20745-20752.

https://scholar.uwindsor.ca/chemistrybiochemistrypub/83

This Article is brought to you for free and open access by the Department of Chemistry and Biochemistry at Scholarship at UWindsor. It has been accepted for inclusion in Chemistry and Biochemistry Publications by an authorized administrator of Scholarship at UWindsor. For more information, please contact scholarship@uwindsor.ca. 


\title{
A Self-Assembled, Low-Cost, Microstructured Layer for Extremely Stretchable Gold Films
}

\author{
Heather L. Filiatrault, R. Stephen Carmichael, Rachel A. Boutette, and Tricia Breen Carmichael*
}

Department of Chemistry and Biochemistry, University of Windsor, Windsor, Ontario, Canada N9B3P4 E-mail: tbcarmic@uwindsor.ca

\begin{abstract}
We demonstrate a simple, low-cost, and green approach to deposit a microstructured coating on the silicone elastomer polydimethylsiloxane (PDMS) that can be coated with gold to produce highly stretchable and conductive films. The microstructured coating is fabricated using an aqueous emulsion of poly(vinyl acetate) (PVAc): common, commercially available white glue. The aqueous glue emulsion self-assembles on the PDMS surface to generate clustered PVAc globules, which can be conformally coated with gold. The microstructured surface provides numerous defect sites that localize strain when the structure is stretched, resulting in the initiation of numerous microcracks. As the structure is further elongated, the microcracks interact with one another, preventing long-range crack propagation and thus preserving the conduction pathway. The resistance of PDMS/glue/gold structures remains remarkably low (23x the initial resistance) up to $65 \%$ elongation, making these structure useful as stretchable interconnects. Decreasing the concentration of the PVAc aqueous emulsion reduces the density of defect sites of the microstructure, which increases the change in resistance of the gold films with stretching. In this way, we can tune the resistance changes of the PDMS/glue/gold structures and increase their sensitivity to strain. We demonstrate the use of these structures as wearable, soft strain sensors.
\end{abstract}


Keywords stretchable electronics, stretchable interconnects, strain sensors, conductors, wearable electronics

\section{Introduction}

The fabrication of electronic devices on soft, elastomeric substrates has opened the way to entirely new and exciting applications for electronics. ${ }^{1-5}$ Stretchable light-emitting devices ${ }^{6-9}$ are the building blocks for foldable and expandable display screens, electronics-integrated clothing, and wallpaper-like lighting. Other devices can be worn on the body, such as smart surgical gloves that sense temperature and electrical potential $^{10}$ and wearable health monitors that acquire and wirelessly transmit electrophysiological data. ${ }^{11}$ A step further leads to devices that can be integrated inside the body, such as stretchable balloon catheters that can "map and zap" damaged areas of the heart. ${ }^{12}$ These diverse stretchable electronics applications have a common feature: Each application requires robust conductive materials for use as device electrodes and interconnects that maintain electrical conductivity during stretching. Metals are the materials of choice due to their low resistivities $\left(\sim 10^{-8} \Omega \mathrm{m}\right)^{13}$; however, the ability of metal films on elastomers to remain conductive during stretching is limited due to cracks that propagate across the metal film as it is elongated and interrupt the conductive pathway. ${ }^{14-17}$ Here, we describe a simple, low-cost approach to create a microstructured layer on the silicone elastomer polydimethylsiloxane (PDMS) that can be coated with gold. This microstructure provides numerous sites for strain localization when this structure is stretched, resulting in the initiation of numerous microcracks. Interactions between these microcracks prevent long-range crack propagation, which better preserves the conduction pathway at high strains. We demonstrate the use of these extremely stretchable gold films as stretchable device interconnects and wearable strain sensors.

Stretchable conductors are essential to all stretchable electronic devices, where they are used as device interconnects and contacts. The challenge with developing useful stretchable conductors is the trade-off that often occurs between stretchability and high conductivity. For example, films of carbon nanotubes, ${ }^{18}$ graphene, ${ }^{19}$ or conducting polymers on $\mathrm{PDMS}^{20,21}$ retain their conductivity to high elongations, but the 
inherently high resistivity of these systems limits their utility as circuit interconnects and electrodes. The obvious way to increase the conductivity is to use metal films, which are ubiquitous as conductive elements in conventional rigid devices and circuits. Freestanding metal films typically fracture at a few percent tensile strain due to necking, unless there is a high rate sensitivity or a lack of imperfections in the metal. ${ }^{22}$ Adhering a metal film to a compliant substrate such as PDMS can also suppress the strain localization that is responsible for necking and rupture of freestanding films. ${ }^{23,24}$ Gold films deposited on PDMS, however, can adopt two possible topographies that strongly affect how strain is localized in the film: The first topography, which consists of a buckled surface, initially exhibits a low resistance that increases linearly up to a critical strain (typically $10-20 \%) .{ }^{14}$ The resistance increases dramatically beyond this critical strain due to the propagation of long, straight cracks that interrupt the conduction pathway. The second type of topography consists of tribranched, Y-shaped microcracks. ${ }^{16,25}$ The initial resistance of this film type is higher than that of the buckled type, but these structures can remain conductive up to $60 \%$ strain due to the formation of a network of microcracks that preserves an interconnected network of gold ligaments for electrical conduction. An understanding of the conditions necessary to selectively prepare gold films with either buckled or tribranch cracked topographies puzzled researchers until a recent report by Graudejus et al. revealed the complexity of this fabrication process by elucidating a number of pertinent variables, such as the gold film thickness, strength of adhesion of the gold to PDMS, deposition temperature, adhesion layer thickness, etc., that operate together to determine the gold film topography. ${ }^{17}$

The complex interplay of the fabrication parameters that determines the topography of gold films deposited on PDMS, and hence the elongation at electrical failure, makes the implementation of these gold/PDMS structures as interconnects and contacts in stretchable devices challenging. Thus, there is a need for gold films on elastomers that reproducibly remain conductive at high elongations. To date, research has largely focused on altering the configuration of gold films by either stretching the PDMS substrate before gold deposition and then releasing the pre-stretch to configure the gold film into a wavy 
structure, or lithographically patterning gold films into serpentine wire patterns on PDMS. Stretching the former structures flattens the waves, and conductivity persists to the pre-stretch elongation, up to $100 \%{ }^{25-}$ 27 The latter structures act like helical springs when the gold/PDMS structure is elongated, which allows conductivity to persist to elongations of $70-100 \%$ depending on the dimensions of the serpentine. ${ }^{28,29}$ Both types of stretchable gold structures are useful as device interconnects, but not as electrodes due to the out-of-plane structure of prestretched gold films and the limited surface area of serpentines. A different approach to achieving highly stretchable gold films that has received less attention uses topographical features on the surface of the underlying PDMS to provide numerous sites for strain localization to intentionally produce a network of microcracks - similar to gold films on flat PDMS with the tribranch-microcracked topography - that preserve multiple pathways for conduction. Examples include geometrical patterns of topographical features such as nanoscale pyramids ${ }^{30}$ or micron-scale circular pillars ${ }^{31}$ fabricated by molding PDMS against lithographically-fabricated masters. The reliance on lithography, however, is incompatible with high-volume, lightweight electronics due to its inherently high cost. To avoid the use of lithography, Lambricht et al. described the use of sandblasted polycarbonate substrates as masters to prepare PDMS substrates with rough surfaces. ${ }^{32}$ The random peaks and valleys on the PDMS surface provide numerous sites for strain localization to initiate microcracks. Thin gold films (80 nm thickness) on rough PDMS substrates remain conductive to $40 \%$ elongation, at which point the resistance of the film $(\mathrm{R})$ reaches $1000 \mathrm{x}$ the initial resistance $\left(\mathrm{R}_{0}\right)$. Thicker gold films $(500 \mathrm{~nm}$ thickness) on these substrates remain conductive to $50 \%$ elongation while retaining an $\mathrm{R} / \mathrm{R}_{0}$ value of 5 .

Here, we report a low-cost, bottom-up, and organic solvent-free approach to create topographical features on the surface of PDMS that provide numerous sites for strain localization in a gold film deposited on the surface. Our method uses low-cost spin-coating to deposit an aqueous emulsion of poly(vinyl acetate) (PVAc) - common, commercially available white glue diluted in water - onto an oxidized PDMS surface. The PVAc emulsion self-assembles on the PDMS surface to form a random array of clustered PVAc globules. When coated with gold, these structures remain remarkably conductive up to $65 \%$ 
elongation, with an increase in resistance to only $23 \mathrm{x}$ the initial resistance of the film. We further demonstrate that the resistance change can be tuned by altering the concentration of the glue emulsion, leading to applications such as stretchable interconnects and soft strain sensors.

\section{Results and Discussion}

White glue is a synthetic thermoplastic adhesive consisting of an emulsion of PVAc in water, along with proprietary additives to stabilize the emulsion. ${ }^{33}$ White glue can be diluted with water, thus avoiding the use of organic solvents, to reduce both the viscosity of the glue and the concentration of colloidal PVAc particles in the suspension. Dark-field optical micrographs of three glue dilutions (1:1, 3:1, and 5:1 v/v water:glue) show the globules suspended in water; the largest visible globules are $\sim 2-3 \mu \mathrm{m}$ in diameter (Figure S1). We spin-coated each of the three formulations onto PDMS substrates that were oxidized for $10 \mathrm{~s}$ in an air plasma to enable wetting to produce three structures referred to as PDMS/glue $1: 1$, PDMS/glue $3: 1$, and PDMS/glue 5:1. Scanning electron microscopy (SEM) and tapping-mode atomic force microscopy (AFM) reveal that the globules of PVAc in the emulsion self-assemble on the PDMS surface to form microstructured coatings (Figure 1). The density of these globules depends on the concentration of glue in the emulsion. At the highest concentration of glue (1:1 v/v water:glue), the surface of the PDMS substrate is homogeneously covered in densely packed glue globules that range in diameter from $\sim 8 \mu \mathrm{m}$ to $<1 \mu \mathrm{m}$ (Figure 1a, b), with a film thickness of $\sim 2-3.5 \mu \mathrm{m}$ (Figure S2a). Increasing the water:glue ratio to $3: 1$ and 5:1 produces surfaces that are not homogeneously coated in glue globules; rather, these surfaces consist of individual and clustered globules of PVAc glue randomly distributed over the PDMS surface (Figure 1c-f). The largest PVAc clusters on PDMS/glue $3: 1$ are $\sim 40 \mu \mathrm{m}$ in diameter and $\sim 3 \mu \mathrm{m}$ in height; on PDMS/glue $5: 1$ the largest clusters are $~ 20 \mu \mathrm{m}$ in diameter and $\sim 2 \mu \mathrm{m}$ in height. The developed interfacial area ratio $\left(\mathrm{S}_{\mathrm{dr}}\right)$ is a useful way to compare surfaces that differ in their spatial and amplitude characteristics, ${ }^{34}$ such as these PDMS/glue surfaces. The $S_{\mathrm{dr}}$ value compares the topographical surface

area to the geometrical area of a sample, and is expressed as the percentage of additional surface area 
contributed by the surface texture. This parameter is sensitive to changes in both the amplitude of the surface features and their spatial arrangement on the surface. For example, surfaces with high amplitude, widely spaced features may actually have a lower $S_{d r}$ value than surfaces with features of lower amplitude that are spaced more closely. We used topographical data from each of the AFM images of PDMS/glue $1: 1$, PDMS/glue $3: 1$, and PDMS/glue $5: 1$ in Figure 1 to determine the $S_{\mathrm{dr}}$ values according to the equation below: ${ }^{34}$

$$
\mathrm{S}_{\mathrm{dr}}=[(\text { topographical surface area })-(\text { geometrical area })] /(\text { geometrical area }) \times 100 \%
$$

The $\mathrm{S}_{\mathrm{dr}}$ value for PDMS/glue $1: 1$ is $23 \%$, consistent with a rough surface bearing finely spaced features. ${ }^{35}$ The $\mathrm{S}_{\mathrm{dr}}$ value decreases to $10 \%$ for PDMS/glue $3: 1$, and further to $4 \%$ for PDMS/glue 5:1, which reflects the increasing spacing between glue globule clusters on these two surfaces, despite the high amplitude of the glue globule clusters. SEM cross sections show that glue $3: 1$ and glue $5: 1$ coatings consist of clusters of PVAc globules separated by regions of bare PDMS (Figure S2b, c). Therefore, we can also compare the three PDMS/glue surfaces in terms of their glue coverage on the surface. Whereas the PVAc globules in the glue $1: 1$ coating cover $100 \%$ of the PDMS surface, we estimate that the coverage of the glue $3: 1$ and glue $5: 1$ coatings is lower, at $\sim 44 \%$ and $\sim 17 \%$, respectively. 

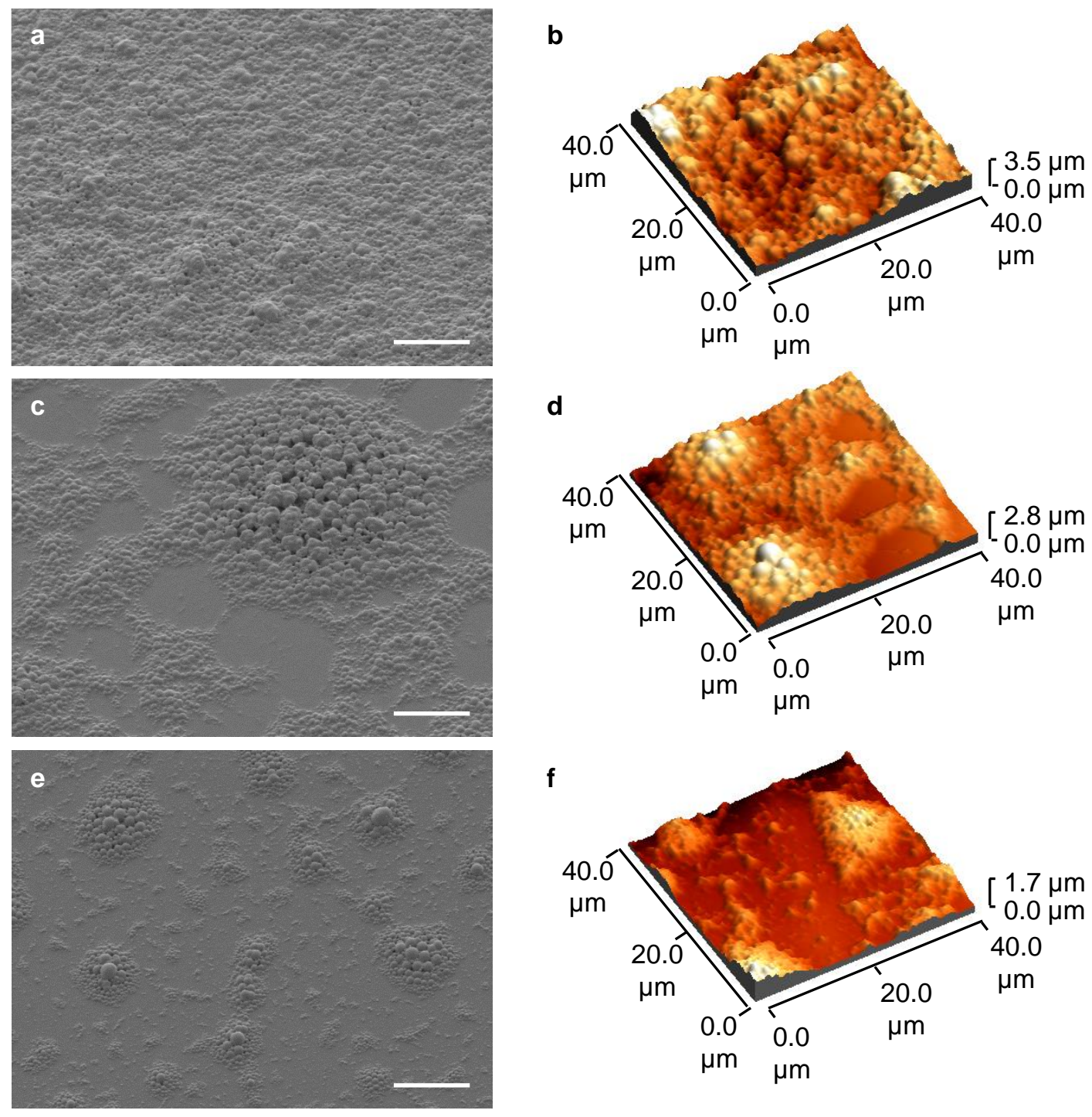

Figure 1. SEM and tapping-mode AFM images of (a, b) PDMS/glue $1: 1 ;(c, d)$ PDMS/glue $3: 1 ;(e, f)$ PDMS/glue5:1. Scale bar $=20 \mu \mathrm{m}$.

The structural differences between the continuous glue $1: 1$ coating and the inhomogeneous glue $3: 1$ and glue 5:1 coatings become relevant when PDMS/glue structures are stretched. Although glue coatings form compliant layers on PDMS due to the rubbery nature of PVAc (the main component of white glue), the Young's modulus of glue (400 MPa for Elmer's glue ${ }^{36} ; 750 \mathrm{MPa}$ for pure PVAc ${ }^{37}$ ) is higher than that of PDMS $(1-2 \mathrm{MPa})^{38}$. All three PDMS/glue structures could be stretched to $60 \%$ elongation without visible delamination; however, optical microscope images reveal that stretching causes the formation of zigzag microscale cracks in the glue ${ }_{1: 1}$ coating due to the high Young's modulus of the continuous glue 
coating (Figure S3a). The random peaks and valleys of the rough glue $1: 1$ surface provide numerous sites for strain localization, which initiates the microcracks that relieve the strain. In contrast, glue $3: 1$ and glue 5:1 coatings do not exhibit cracks (Figure S3b, c); instead, the regions between the PVAc glue islands likely absorb the strain and allow the PVAc clustered globules to behave like rigid islands.

We used an e-beam evaporator to coat PDMS/glue surfaces with a 30 - $\AA$-thick layer of titanium as an adhesion promoter followed by a 250 - $\AA$-thick layer of gold. SEM micrographs show that the metal uniformly coats the surfaces of PDMS/glue $1: 1$, PDMS/glue $3: 1$, and PDMS/glue $5: 1$ to yield gold-coated surfaces with topographical features that are indistinguishable from those of unmetallized PDMS/glue surfaces (Figure S4). The differences in topography caused by the use of three different glue dilutions does not measurably affect the sheet resistance values $\left(R_{s}\right)$ of overlying gold films, which are similar to the $\mathrm{R}_{\mathrm{s}}$ of planar 250 - $\AA$-thick gold films deposited on glass (Table 1). Gold films deposited on the PDMS/glue samples pass the tape test, indicating that the glue layer does not adversely affect the adhesion.

Table 1. Sheet resistance $\left(\mathrm{R}_{\mathrm{s}}\right)$ of 250 - $\AA$-thick-gold films on glass and PDMS/glue substrates.

\begin{tabular}{ll}
\hline Substrate & $\mathrm{R}_{\mathrm{s}}(\Omega / \square)$ \\
\hline Glass & $1.8 \pm 0.1$ \\
PDMS/glue $_{1: 1}$ & $2.5 \pm 0.2$ \\
PDMS/glue $_{3: 1}$ & $2.4 \pm 0.2$ \\
PDMS/glue $_{5: 1}$ & $1.9 \pm 0.2$
\end{tabular}

The topography of the glue layer in PDMS/glue/gold structures profoundly changes how cracks initiate and propagate with stretching compared to gold films deposited on smooth PDMS without a glue layer (PDMS/gold). When metal films adhered to compliant substrates are stretched, cracks initiate at defect sites in the metal film. ${ }^{15,39}$ The cracks propagate and lengthen to relieve strain as the sample is elongated further, unless they approach another crack. At this point, the propagating crack enters a relaxed zone where the strain has already been relieved; consequently, the crack stops propagating, resulting in a film 
comprising numerous small cracks rather than fewer long straight cracks. ${ }^{32}$ Optical microscope images of PDMS/gold at 5\% and 25\% strain (Figure 2) demonstrate that smooth PDMS provides few defect sites on the surface for strain localization and crack nucleation. Even at low strains of 5\%, cracks form and propagate through the field of view of a 10x microscope objective (that is, at least $1000 \mu \mathrm{m}$ ) to relieve strain. Although the number of cracks increases with further elongation to $25 \%$ (Figure 2b), these cracks also propagate to relieve strain, forming an array of long and straight cracks. In contrast, optical microscope images of PDMS/glue 1:1/gold, PDMS/glue 3:1/gold, and PDMS/glue 5:1/gold structures taken at 5, 25, and 60\% strain (Figure 3) show that the rough topography of the PVAc glue globules provides numerous sites for strain localization and crack nucleation. There is a rapid increase in the number of cracks as these structures are stretched from 5\% to $60 \%$ strain. The cracks encounter and interact with one another, which prevents their propagation and limits the maximum crack length to $\sim 250 \mu \mathrm{m}$.

The numerous small cracks caused by the topography of PDMS/glue/gold structures furthermore create an interconnected gold film that effectively preserves conduction during linear strain. A comparison of the plots of the change in resistance of PDMS/glue 1:1/gold, PDMS/glue 3:1/gold, PDMS/glue5:1/gold as a function of linear strain (Figure 4) with that of PDMS/gold (Figure 4, inset) show that the use of a glue layer both considerably lowers the change in resistance with stretching and increases the elongation at failure: The resistance of PDMS/gold rises rapidly to 909 times the initial value at $35 \%$ strain. Although the structure can be stretched further without fracturing, after 35\% elongation the gold film becomes nonconductive due to the long, straight cracks that have broken the conductive pathway. In contrast, the interconnected network of short cracks that forms on PDMS/glue/gold structures enables these structures to remain conductive up to $65-70 \%$ elongation, and the $\mathrm{R} / \mathrm{R}_{0}$ to remain $<243$. These structures ultimately fail at $\sim 70 \%$ elongation due to fracture through the PDMS/glue/gold stack that separates the sample into two pieces rather than simply the termination of conductivity. This fracture is likely due to the extension of cracks from the gold layer into the glue layer and PDMS substrate caused by the large mismatch in 
elastic properties (Young's modulus, $E$ ) between gold $(E=79 \mathrm{GPa})^{40}$, glue $(E=400 \mathrm{MPa}$ for white glue $^{36} ; 750 \mathrm{MPa}$ for pure PVAc $\left.{ }^{37}\right)$ and PDMS $(1-2 \mathrm{MPa})^{38} \cdot{ }^{41}$
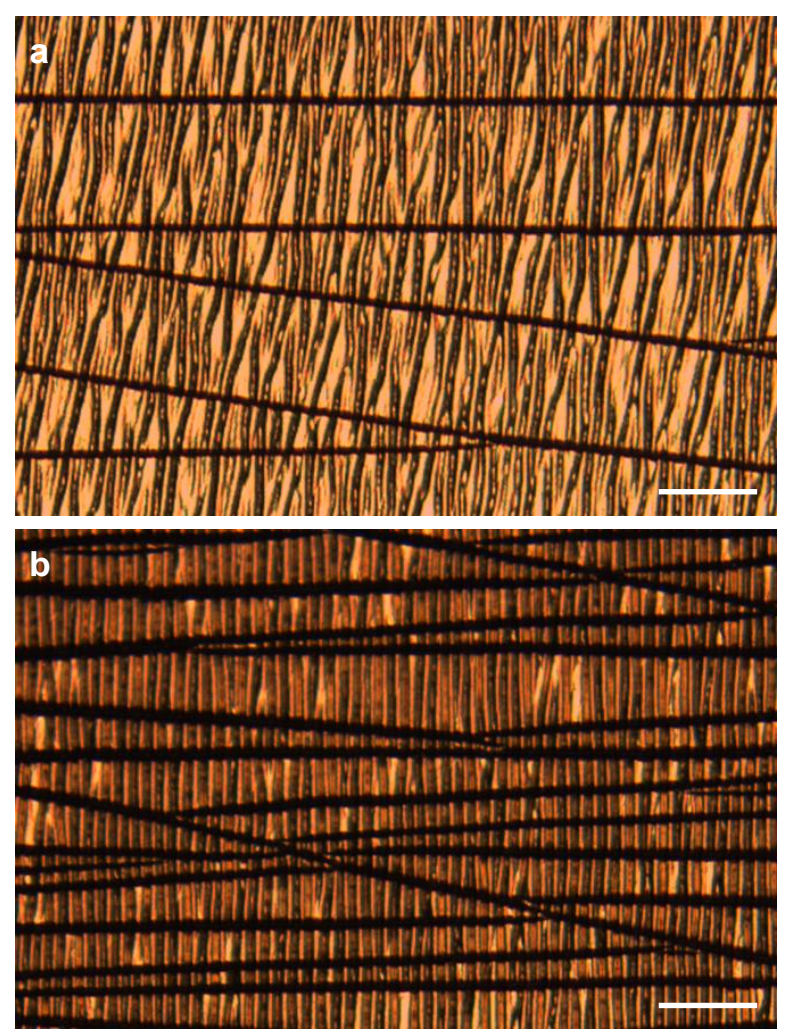

Figure 2. Evolution of cracks on PDMS/gold structures with stretching. Optical images captured at (a) 5\% strain and (b) 25\% strain. The sample was stretched in the vertical direction, which is accompanied by compressive lateral strain that can be observed as buckles parallel to the direction of stretching. Scale $\operatorname{bar}=80 \mu \mathrm{m}$. 

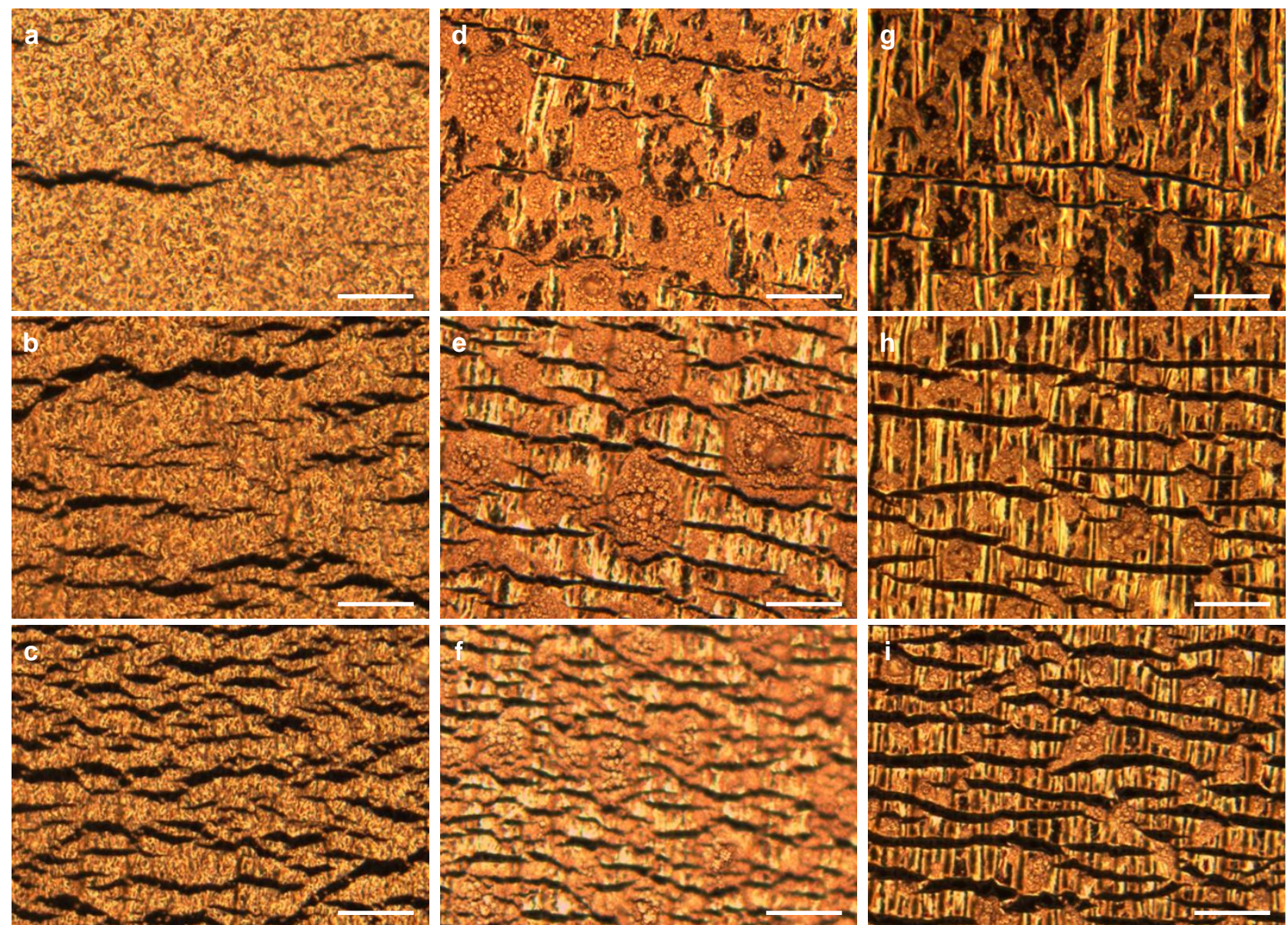

Figure 3. Evolution of cracks on PDMS/glue/gold structures with stretching. Optical images captured at $5 \%$ (top row), $25 \%$ (middle row), and $60 \%$ (bottom row) strain for (a-c) PDMS/glue 1:1/gold; (d-f) PDMS/glue3:1/gold; (g-i) PDMS/glue5:1/gold. In all images, the samples were stretched in the vertical direction, which is accompanied by compressive lateral strain that can be observed as buckles parallel to the direction of stretching. Scale bar $=40 \mu \mathrm{m}$. 


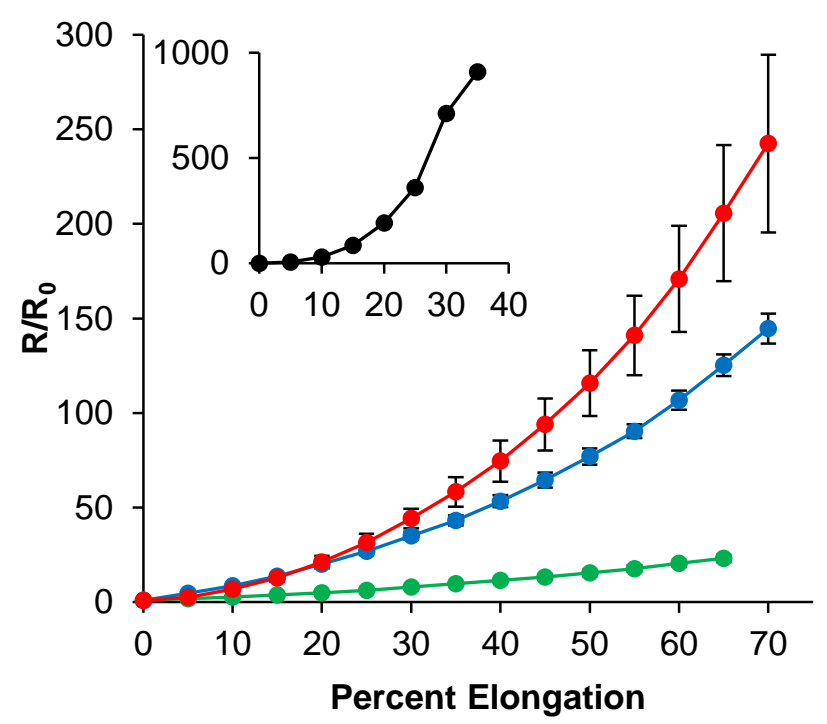

Figure 4. Plot of normalized resistance as a function of linear strain for PDMS/glue 1:1/gold (green), PDMS/glue 3:1/gold (blue), PDMS/glue 5:1/gold (red), and PDMS/gold (inset, data from the best sample due to the high variability of this control).

Figure 4 also demonstrates that the resistance changes of PDMS/glue/gold structures with stretching can be tuned by simply changing the glue concentration used to form the glue layer, which alters both the coverage of glue globules on the PDMS surface and the $S_{\mathrm{dr}}$ value of the surface. The highest glue coverage $(100 \%)$ and $\mathrm{S}_{\mathrm{dr}}$ value (23\%) of PDMS/glue $1: 1 /$ gold structures causes the smallest overall change in resistance, whereas the lowest glue coverage $(\sim 17 \%)$ and $\mathrm{S}_{\mathrm{dr}}$ value $(4 \%)$ of PDMS/glue 5:1/gold structures causes the largest change in resistance. Thus, the most "defective" surface - the surface with the greatest number of defect sites for strain localization and crack initiation - causes the lowest increase in R. To illustrate the relationship between the structures of the gold films resulting from the glue coverage and their electrical function under strain, we correlate the resistance measurements of PDMS/glue $1: 1 /$ gold, PDMS/glue 3:1/gold, PDMS/glue 5:1/gold (Figure 4, with data values provided in Table 2 for reference) with the images of the cracking patterns collected 5, 25, and 60\% strain in Figure 3 . At 5\% strain, the three PDMS/glue/gold structures exhibit a similar increase in resistance $\left(\mathrm{R} / \mathrm{R}_{0}<5\right)$. Despite the similarity in resistance change, however, there are differences in the way cracks initially form in these structures. 
PDMS/glue/gold structures all bear cracks of various lengths, the longest of these being $\sim 100 \mu \mathrm{m}$ (Figure 3a, d, and g). On PDMS/glue 3:1/gold and PDMS/glue 5:1/gold, the cracks appear to initiate at the edges of the isolated glue globule clusters. These cracks propagate through the relatively defect-free smooth regions between clusters, and then terminate at the edge of another glue globule cluster. This apparent guiding of how the cracks propagate results in cracks that are rather straight. In contrast, the cracks on PDMS/glue $1: 1 /$ gold take on a more irregular, jagged shape due to the consistently rough topography of this surface, which provides numerous sites for strain localization. The differences in the initial crack formation at $5 \%$ elongation begins to differentiate the $\mathrm{R} / \mathrm{R}_{0}$ values of PDMS/glue $1: 1 /$ gold from PDMS/glue 3:1/gold and PDMS/glue5:1/gold when the elongation is increased to 25\%. Here, PDMS/glue 1:1/gold retains a low $\mathrm{R} / \mathrm{R}_{0}$ value of 6.4 , whereas the $\mathrm{R} / \mathrm{R}_{0}$ values of PDMS/glue $3: 1 /$ gold and PDMS/glue 5:1/gold increase to 26.9 and 31.6, respectively. Although all of these structures exhibit an increase in the number of cracks to relieve strain, the main difference is that there is no appreciable lengthening of the cracks on PDMS/glue $1: 1 /$ gold (Figure 3b), whereas the cracks on PDMS/glue $3: 1 /$ gold and PDMS/glue 5:1/gold are substantially lengthened to $>200 \mu \mathrm{m}$. This lengthening of the cracks creates a tortuous path for current flow, which may account for the greater increase in $R / R_{0}$. At $60 \%$ strain, the three sample types are all dissimilar: The resistance of PDMS/glue 1:1/gold remains rather low $\left(\mathrm{R} / \mathrm{R}_{0}=20.5\right)$, the resistance of PDMS/glue $3: 1 /$ gold increases to $\mathrm{R} / \mathrm{R}_{0}=106.8$, and the resistance of PDMS/glue 5:1/gold increases dramatically to $\mathrm{R} / \mathrm{R}_{0}=170.9$. Differences in the crack dimensions account for the observed differences in resistance change. Although the most conductive structure, PDMS/glue 1:1/gold, develops new, small cracks to relieve the strain, the structure preserves $\sim 30$ - $\mu$ m-wide strips of gold between the cracks that undoubtedly help maintain conductivity (Figure 3c). The preservation of these gold strips can be attributed to the numerous cracks in the gold film that interact with one another, limiting the propagation length: A propagating crack has a high probability of entering into the relaxed zone near another crack where the strain has already been relieved, thus halting propagation. Similarly, PDMS/glue 3:1/gold also develops small, new cracks; however, the continuous 
gold strips separating the cracks are narrower $(\sim 10-15 \mu \mathrm{m})$, which increases the resistance (Figure 3f). PDMS/glue 5:1/gold is distinguished from the other two structure types by long cracks that become longer and wider in response to the strain (Figure 3i). At $60 \%$ strain, these cracks can reach $\sim 250 \mu \mathrm{m}$ in length, with a typical width of $\sim 50 \mu \mathrm{m}$. These long, wide cracks dominate the gold structure, leaving a limited conductive pathway through the film.

Table 2: Change in resistance of PDMS/glue/gold structures at 5, 25, and 60\% elongation.

\begin{tabular}{cccc} 
& \multicolumn{3}{c}{$\mathrm{R} / \mathrm{R}_{0}$} \\
Glue Formulation & $5 \%$ & $25 \%$ & $60 \%$ \\
\cline { 2 - 4 } & 1.9 & 6.4 & 20.5 \\
$3: 1$ & 4.8 & 26.9 & 106.8 \\
$5: 1$ & 2.6 & 31.6 & 170.9
\end{tabular}

The high conductivity of PDMS/glue 1:1/gold that persists with stretching to $65 \%$ elongation makes these films suitable as interconnects in stretchable electronic devices. As a proof-of-concept demonstration, we constructed circuits consisting of a source-measure unit and a green LED connected in series with a $1.5 \mathrm{~cm} \times 2.5 \mathrm{~cm}$ stretchable gold interconnect, which acts as a variable resistor $\left(\mathrm{R}_{\mathrm{var}}\right)$; that is, the resistance of the interconnect increases as it is elongated (Figure 5a). The LED demonstrates a voltage drop of $2.2 \mathrm{~V}$ when biased with a current of $20 \mathrm{~mA}$. As the interconnect is stretched, the voltage required to drive the LED increases due to the increasing voltage drop across the gold film. We measured the total voltage drop $\left(\mathrm{V}_{\mathrm{T}}\right)$ of the circuit as the interconnect was elongated from $0 \%-50 \%$, and plotted $\mathrm{V}_{\mathrm{T}}$ as a function of interconnect elongation for interconnects fabricated from PDMS/glue 1 /1/gold and, for comparison, PDMS/glue 3:1/gold (Figure 5b). At $0 \%$ strain, $\mathrm{V}_{\mathrm{T}}$ is $2.4 \mathrm{~V}$ for both PDMS/glue $1: 1 /$ gold and PDMS/glue $3: 1 /$ gold. $\mathrm{V}_{\mathrm{T}}$ of the PDMS/glue $1: 1 /$ gold interconnected circuit increases to $5.0 \mathrm{~V}$ at $50 \%$ strain. 
In contrast, $\mathrm{V}_{\mathrm{T}}$ increases more quickly for the PDMS/glue 3:1/gold interconnect, and reaches $6.5 \mathrm{~V}$ at $50 \%$ elongation. The steeper slope of the $\mathrm{V}_{\mathrm{T}}$ versus percent elongation for PDMS/glue 3:1/gold is consistent with the plots of $\mathrm{R} / \mathrm{R}_{0}$ versus elongation presented in Figure 4.

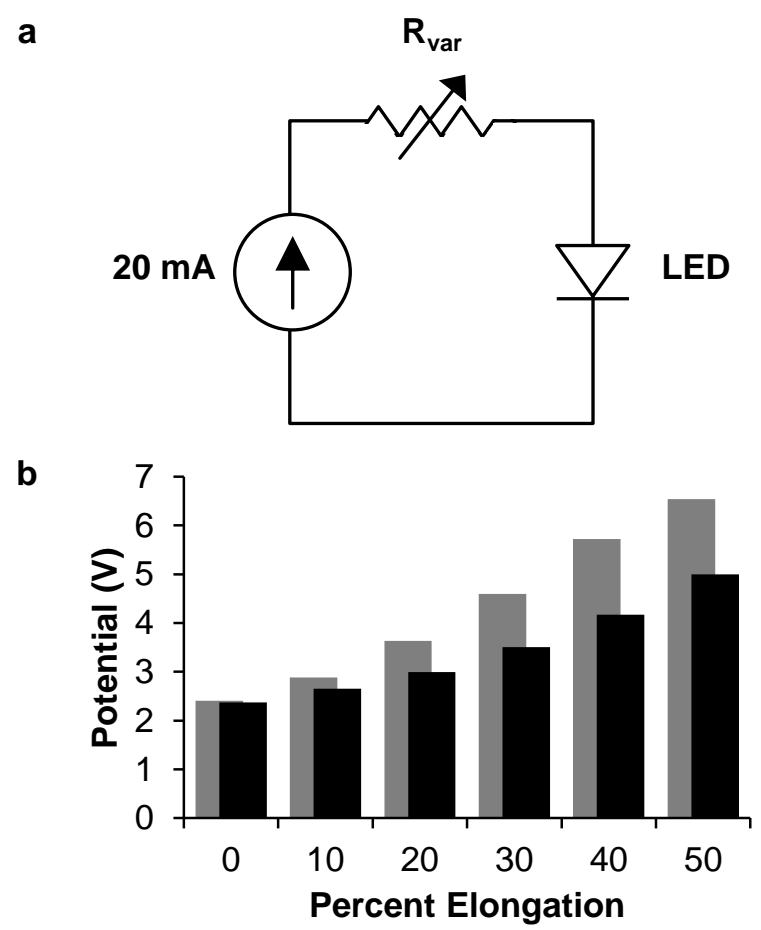

Figure 5. Demonstration of PDMS/glue 1:1/gold and PDMS/glue $3: 1 /$ gold as stretchable interconnects. (a) Circuit diagram; (b) Plot of $\mathrm{V}_{\mathrm{T}}$ as a function of percent elongation for PDMS/glue $1: 1$ /gold (black bars) and PDMS/glue $3: 1 /$ gold (grey bars).

The sensitivity of PDMS/glue $3: 1 /$ gold structures to changes in strain makes them potentially useful as soft strain sensors. Strain sensors detect mechanical deformation through a change in electrical resistance. Metal foils patterned on a flexible plastic backing are a common gauge type. The gauge is mounted on the object to be monitored, and deformation of the object translates into a change in electrical resistance of the gauge. The sensitivity of the gauge to mechanical deformation is quantified as the gauge factor (GF), which relates the fractional change in resistance to the mechanical strain:

$$
\mathrm{GF}=\frac{\Delta \mathrm{R} / \mathrm{R}_{0}}{\varepsilon}
$$


Conventional metal foil strain sensors have a GF of 2 to 5 , and can stretch to a maximum of $5 \%$ before electrical failure. ${ }^{42}$ Biomechanics, physiology, kinesiology, and robotics applications, however, require soft strain sensors that can conform easily to the curves of the human body or over robotic joints to allow unimpeded motion, while at the same time being capable of detecting large strains. ${ }^{43}$ Metals deposited on elastomers - a soft version of the classic metal strain gauge - are a possible solution. Although the limited stretchability of gold films on PDMS hinders their use as human motion strain sensors, PDMS/glue 3:1/gold structures are highly stretchable, compatible with the soft nature of the human body, and sensitive to changes in strain. We fabricated a simple PDMS/glue $3: 1 /$ gold strain sensor as a proof-ofconcept by attaching a $1.5 \mathrm{~cm}$ x $3.5 \mathrm{~cm}$ PDMS/glue $3: 1 /$ gold structure to an adhesive bandage and attaching electrical contacts using conductive silicone paste. The bandage was then adhered along the length of a thumb (Figure 6a). We applied a voltage of $0.5 \mathrm{~V}$ and recorded the change in resistance as the thumb was bent (Figure 6b) and straightened repetitively. Bending the thumb fully resulted in a tenfold increase in resistance (Figure 6c); the resistance returned to the initial value upon straightening of the thumb. The initial resistance was recovered after six cycles of the bending/straightening process. The action of these thumb-mounted strain sensors can also be visualized using a series of LEDs interfaced by an Arduino singleboard microcontroller (Video S1). We used the resistance vs strain data presented in Figure 4 to elucidate three key features of PDMS/glue 3 :1/gold strain sensors: First, the tenfold increase in resistance produced by bending the thumb corresponds to a strain of approximately $15 \%$. To assess the durability of our strain sensors at this strain value, we measured the resistance after 10 cycles of $15 \%$ elongation and repeated to a total of 100 strain cycles (Figure S5). Throughout the testing, the resistance remained remarkably constant, increasing to a maximum $\mathrm{R} / \mathrm{R}_{0}$ value of 1.2 . Second, these structures are capable of detecting much larger strains as they remain conductive up to $70 \%$ elongation, which suggests that they could be used to detect a wide range of human or robotic motion. Third, the output of PDMS/glue $3: 1 /$ gold strain sensors is nonlinear in resistance versus strain, similar to semiconductor strain sensors. ${ }^{44,45}$ The GF thus is not constant as the strain takes place; rather, it increases from 96 at $5 \%$ strain to 201 at $70 \%$ strain. 

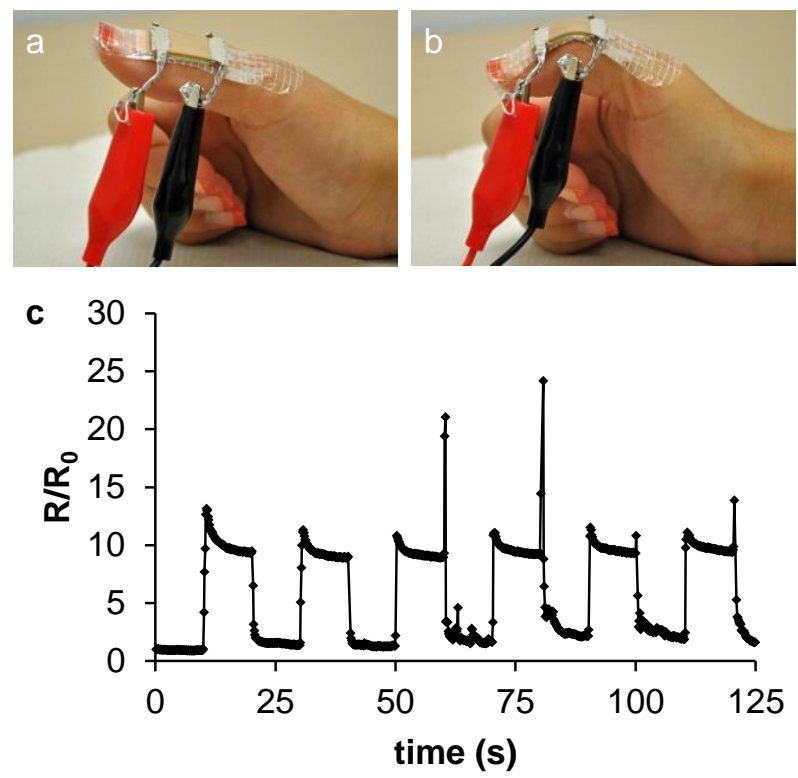

Figure 6. Demonstration of PDMS/glue 3:1/gold as a wearable strain sensor. Photograph of the sensor mounted on a human thumb in (a) straightened (unstrained) and (b) bent (strained) positions. (c) Plot of normalized resistance as a function of time corresponding to six bending/straightening cycles.

\section{Conclusions}

We have demonstrated a simple, low-cost, and green approach to fabricate microstructured PDMS surfaces that produce highly stretchable and conductive gold films. The PVAc glue used to create the microstructure is low cost, commercially available, and can be deposited from aqueous solution to avoid the use of organic solvents. By changing the concentration of the PVAc globules in the emulsion, we alter the topography, spatial distribution, and coverage of the glue globules on the PDMS surface. In this way, we can tune the change in resistance in the gold films deposited on the surface when they are subjected to strain. High concentrations of glue (PDMS/glue $\left.{ }_{1: 1}\right)$ result in small changes in resistance making these wires ideal as stretchable interconnects. Gold films deposited onto films fabricated using more dilute glue solutions, such as PDMS/glue $3: 1$, have higher sensitivities, which make them potentially useful as soft, stretchable versions of conventional metal foil strain gauges. These soft sensors are highly 
conformable and can detect strains up to $70 \%$, which makes them good candidates for low-cost motion detection across both human and robotic joints.

\section{Methods}

Poly(dimethylsiloxane) (PDMS) (Sylgard 184) was obtained from Dow Corning, Midland, MI. All other chemicals were obtained from Sigma-Aldrich and were used as received. White glue (Elmer's GlueAll) was obtained from Elmer's Products, Inc (Westerville, Ohio).

Preparation of PDMS/Glue/Gold Structures PDMS substrates were prepared by casting a 10:1 w:w ratio of prepolymer:curing agent against polystyrene Petri dishes and curing at $60{ }^{\circ} \mathrm{C}$ for $1 \mathrm{~h}$, and then treated with air plasma at medium discharge setting for 10 seconds at an air pressure of 10 psig (flow rate of $32 \mathrm{~mL} / \mathrm{min}$ ). (Harrick Plasma PDC32-G coupled to a PlasmaFlo gas flow mixer). Glue solutions of Elmer's Glue-All diluted in water ( 1:1 v/v water:glue, 3:1 v/v water:glue and 5:1 v/v water:glue) were spin-coated on the oxidized PDMS substrates at $1000 \mathrm{rpm}$ for 1 minute followed by $2000 \mathrm{rpm}$ for 1 minute (Laurell Technologies WS-400A-6NPP). We estimated the surface coverage of the glue dilutions on PDMS by calculating the area occupied by clusters measuring $\geq 10 \mu \mathrm{m}$ in diameter in a $500 \mathrm{x}$ magnification SEM image with a total area of $150 \mu \mathrm{m} \times 150 \mu \mathrm{m}$. An e-beam evaporator was used to deposit $30 \AA$ titanium, followed by $250 \AA$ gold, onto PDMS/glue substrates at a rate of $0.3 \AA / s$ and $1 \AA / \mathrm{s}$ respectively, under high vacuum $\left(10^{-6} \mathrm{mbar}\right)$.

Strain Sensor Fabrication PDMS/glue 3:1/gold structures were mounted to a Band-Aid using PDMS pre-polymer as the adhesive, followed by curing at $60{ }^{\circ} \mathrm{C}$ for one $\mathrm{h}$. Connectors were fabricated by gluing strips of aluminum foil to the ends of the PDMS/glue $3: 1 /$ gold structures using a conductive silicone paste (Effective Shielding, West Chester PA).

Characterization Optical characterization was performed using an Olympus BX51 microscope equipped with an Olympus Q-Color3 digital camera. A micro-vice stretcher (S. T. Japan, USA, Inc.) was mounted to the microscope stage and samples were clamped in the stretcher to obtain microscope images 
of stretched samples. AFM images were obtained using the dynamic force mode of a Park Systems XE100 AFM (Surface Science Western, London, ON, Canada). Images were collected from three spots on each sample in an area of $40 \mu \mathrm{m} \times 40 \mu \mathrm{m}$. Images were processed using WSxM 5.0 Develop 1.0 software. ${ }^{46}$ SEM images were obtained using a Hitachi S-4500 scanning electron microscope (Surface Science Western, London, ON, Canada). Electrical characterization was performed using a Keithley 2601A source meter. Gallium-indium eutectic (EGaIn) ( 0.01 mL) was first deposited by syringe to the corners (for sheet resistance measurements) or ends (for resistance measurements) of the gold surface to facilitate electrical contact. Data sets consisted of a minimum of three samples, and the average was reported. For electrical measurements under strain, samples were clamped in a micro-vice stretcher (S.T. Japan, USA, Inc.) and the resistance was measured at 5\% increments of strain.

Supporting Information Available: Arduino code, optical micrographs of glue emulsions, SEM cross sections of PDMS/glue structures, optical micrographs of stretched PDMS/glue structures, SEM images of PDMS/glue/gold surfaces, plot of normalized resistance of PDMS/glue $3: 1 /$ gold with $15 \%$ strain cycles. This material is available free of charge via the Internet at http://pubs.acs.org.

\section{Acknowledgements}

This research was supported by the National Sciences and Engineering Research Council of Canada (NSERC). H. L. F. is grateful for the award of an NSERC post-graduate doctoral scholarship (CGSD). We thank Brad Kobe and Rebecca Jacklin at Surface Science Western for scanning electron microscopy, and Dr. Heng-Yong Nie at Surface Science Western for atomic force microscopy. 


\section{References}

(1) Bauer, S.; Bauer-Gogonea, S.; Graz, I.; Kaltenbrunner, M.; Keplinger, C.; Schwodiauer, R. $25^{\text {th }}$ Anniversary Article: A Soft Future: From Robots and Sensor Skin to Energy Harvesters. Adv. Mater. 2013, 26, 149-162.

(2) Rogers, J. A.; Someya, T.; Huang, Y. Materials and Mechanics for Stretchable Electronics. Science 2010, 327, 1603-1607.

(3) Sekitani, T.; Someya, T. Stretchable, Large-Area Organic Electronics. Adv. Mater. 2010, 22, 2228-2246.

(4) Kim, D. -H.; Xiao, J.; Song, J.; Huang, Y.; Rogers, J. A. Stretchable, Curvilinear Electronics Based on Inorganic Materials. Adv. Mater. 2010, 22, 2108-2124.

(5) Rogers, J. A.; Huang, Y. A Curvy, Stretchy Future for Electronics. Proc. Natl. Acad. Sci. 2009, 106, 10875-10876.

(6) Park, S. -I.; Xiong, Y.; Kim, R. -H.; Elvikis, P.; Meitl, M.; Kim, D. -H.; Wu, J.; Yoon, J.; Yu, C. -J.; Liu, Z.; Huang, Y.; Hwang, K. -C.; Ferreira, P.; Li, X.; Choquette, K.; Rogers, J. A. Printed Assemblies of Inorganic Light-Emitting Diodes for Deformable and Semitransparent Displays. Science 2009, 325, 977-981.

(7) Sekitani, T.; Nakajima, H.; Maeda, H.; Takanori, F.; Aida, T.; Hata, K.; Someya, T. Stretchable Active-Matrix Organic Light-Emitting Diode Display Using Printable Elastic Conductors. Nature Mater. 2009, 8, 494-499.

(8) Liang, J.; Li, L.; Niu, X.; Yu, Z.; Pei, Q. Elastomeric Polymer Light-Emitting Devices and Displays. Nature Photon. 2013, 7, 817-824.

(9) Filiatrault, H. L.; Porteous, G. C.; Carmichael, R. S.; Davidson, G. J. E.; Carmichael, T. B. Stretchable Light-Emitting Electrochemical Cells Using and Elastomeric Emissive Material. Adv. Mater. 2012, 24, 2673-2678.

(10) Ying, M.; Bonifas, A. P.; Lu, N.; Su, Y.; Li, R.; Cheng, H.; Ameen, A.; Huang, Y.; Rogers, J. A. Silicon Nanomembranes for Fingertip Electronics. Nanotechnology 2012, 23 (34), 344004.

(11) Xu, S.; Zhang, Y.; Jia, L.; Mathewson, K. E.; Jang, K. I.; Kim, J.; Fu, H.; Huang, X.; Chava, P.; Wang, R.; Bhole, S.; Wang, L.; Na, Y. J.; Guan, Y.; Flavin, M.; Han, Z.; Huang, Y.; Rogers, J. A. Soft Microfluidic Assemblies of Sensors, Circuits, and Radios for the Skin. Science 2014, 344, 70-74.

(12) Kim, D. H.; Lu, N. S.; Ghaffari, R.; Kim, Y. S.; Lee, S. P.; Xu, L. Z.; Wu, J. A.; Kim, R. H.; Song, J. Z.; Liu, Z. J.; Viventi, J.; de Graff, B.; Elolampi, B.; Mansour, M.; Slepian, M. J.; Hwang, S.; Moss, J. D.; Won, S. M.; Huang, Y. G.; Litt, B.; Rogers, J. A. Materials for Multifunctional Balloon Catheters with Capabilities in Cardiac Electrophysiological Mapping and Ablation Therapy. Nature Mater. 2011, 10, 316-323.

(13) Cutnell, J. D.; Johnson, K. W. Physics, $9^{\text {th }}$ ed; John Wiley \& Sons: New York, 2012.

(14) Lacour, S. P.; Wagner, S.; Huang, Z.; Suo, Z. Stretchable Gold Conductors on Elastomeric Substrates. Appl. Phys. Lett. 2003, 82, 2404-2406. 
(15) Teng, L.; Huang, Z.; Suo, Z.; Lacour, S. P.; Wagner, S. Stretchability of Thin Metal Films on Elastomer Substrates. Appl. Phys. Lett. 2004, 85, 3435-3437.

(16) Lacour, S. P.; Chan, D.; Wagner, S.; Li, T.; Suo, Z. Mechanisms of Reversible Stretchability of Thin Metal Films on Elastomeric Substrates. Appl. Phys. Lett. 2006, 88, 204103.

(17) Graudejus, O.; Görrn, P.; Wagner, S. Controlling the Morphology of Gold Films on Poly(dimethylsiloxane). ACS Appl. Mater. Interfaces 2010, 2, 1927-1933.

(18) Cao, Q.; Rogers, J. A. Ultrathin Films of Single-Walled Carbon Nanotubes for Electronics and Sensors: A Review of Fundamental and Applied Aspects. Adv. Mater. 2009, 21, 29-53.

(19) Kim, K. S.; Zhao, Y.; Jang, H.; Lee, S. Y.; Kim, J. M.; Ahn, J. H.; Kim, P.; Choi, J. Y.; Hong, B. H. Large-scale Pattern Growth of Graphene Films for Stretchable Transparent Electrodes. Nature 2009, 457, 706-710.

(20) Lipomi, D. J.; Lee, J. A.; Vosgueritchian, M.; Tee, B. C. K.; Bolander, J. A.; Bao, Z. Electronic Properties of Transparent Conductive Films of PEDOT:PSS on Stretchable Substrates. Chem. Mater. 2012, 24 (2), 373-382.

(21) Vosgueritchian, M.; Lipomi, D. J.; Bao, Z. Highly Conductive and Transparent PEDOT:PSS Films with a Fluorosurfactant for Stretchable and Flexible Transparent Electrodes. Adv. Funct. Mater. 2012, 22, 421-428.

(22) Pineau, A.; Benzerga, A. A.; Pardoen, T. Failure of Metals III. Fracture and Fatigue of Nanostructured Metallic Materials. Acta Mater. in press. doi:10.1016/j.actamat.2015.07.049

(23) Li, T.; Suo, Z. Deformability of Thin Metal Films on Elastomer Substrates. Int. J. Solids Struct. 2006, 43, 2351-2363.

(24) Li, T.; Huang, Z. Y.; Xi, Z. C.; Lacour, S. P.; Wagner, S.; Suo, Z. Delocalizing Strain in a Thin Metal Film on a Polymer Substrate. Mech. Mater. 2005, 37, 261-273.

(25) Lacour, S. P.; Jones, J.; Wagner, S.; Li, T.; Suo, Z. Stretchable Interconnects for Elastic Electronic Surfaces. Proc. IEEE 2005, 93, 1459-1467.

(26) Jones, J.; Lacour, S. P.; Wagner, S.; Suo, Z. Stretchable Wavy Metal Interconnects. J. Vac. Sci. Technol., A 2004, 22, 1723-1725.

(27) Gorrn, P.; Cao, W.; Wagner, S., Isotropically Stretchable Gold Conductors on Elastomeric Substrates. Soft Matter 2011, 7 (16), 7177-7180.

(28) Brosteaux, D.; Axisa, F.; Gonzalez, M.; Vanfleteren, J. Design and Fabrication of Elastic Interconnections for Stretchable Electronic Circuits. IEEE Electron Device Lett. 2007, 28, $552-554$.

(29) Gray, D. S.; Tien, J.; Chen, C. S. High-Conductivity Elastomeric Electronics Adv. Mater. 2004, 16, 393-397.

(30) Mandlik, P.; Lacour, S. P.; Li, J. W.; Chou, S. Y.; Wagner, S. Fully Elastic Interconnects on Nanopatterned Elastomeric Substrates. IEEE Electron Device Lett. 2006, 27, 650-652. 
(31) Robinson, A. P.; Minev, I.; Graz, I. M.; Lacour, S. P. Microstructured Silicone Substrate for Printable and Stretchable Metallic Films. Langmuir 2011, 27, 4279-4284.

(32) Lambricht, N.; Pardoen, T.; Yunus, S. Giant Stretchability of Thin Gold Films on Rough Elastomeric Substrates. Acta Mater. 2013, 61, 540-547.

(33) Chen, Y. -H.; Yaung, J. -F. A Polymer in Everyday Life: The Isolation of Poly(vinyl alcohol) from Aqueous PVA Glues. J. Chem. Educ. 2006, 83, 1534-1536.

(34) Loberg, J.; Mittisson, I.; Hansson, S.; Ahlberg, E. Characterisation of Titanium Dental Implants I: Critical Assessment of Surface Roughness Parameters. Open Biomater. J. 2010, $2,18-35$.

(35) Dong, W. P.; Sullivan, P. J.; Stout, K. J. Comprehensive Study of Parameters for Characterizing Three-Dimensional Surface Topography IV: Parameters for Characterizing Spatial and Hybrid Properties. Wear 1994, 178, 45-60.

(36) Tseytlin, Y. M., Structural Synthesis in Precision Elasticity; Springer: New York, 2006.

(37) Khan, U.; May, P.; Porwal, H.; Nawaz, K.; Coleman, J. N. Improved Adhesive Strength and Toughness of Polyvinyl Acetate Glue on Addition of Small Quantities of Graphene. ACS Appl. Mater. Interfaces 2013, 5, 1423-1428.

(38) Choi, K. M.; Rogers, J. A. A Photocurable Poly(dimethylsiloxane) Chemistry Designed for Soft Lithographic Molding and Printing in the Nanometer Regime. J. Am. Chem. Soc. 2003, 125, 4060-4061.

(39) Lu, N.; Wang, X.; Suo, Z.; Vlassak, J. Metal Films on Polymer Substrates Stretched Beyond 50\%. Appl. Phys. Lett. 2007, 91, 221909.

(40) Salvadori, M. C.; Vaz, A. R.; Melo, L. L.; Cattani, M. Nanostructured Gold Thin Films: Young Modulus Measurement. Surf. Rev. Lett. 2003, 10, 571-575.

(41) Douville, N. J.; Li, Z.; Takayama, S.; Thouless, M. D. Fracture of Metal Coated Elastomers. Soft Matter 2011, 7, 6493-6500.

(42) Window, A. L., Strain Gauge Technology; Springer: Netherlands 1992.

(43) Lu, N.; Lu, C.; Yang, S.; Rogers, J. Highly Sensitive Skin-Mountable Strain Gauges Based Entirely on Elastomers. Adv. Funct. Mater. 2012, 22, 4044-4050.

(44) Smith, C. S. Piezoresistance Effect in Germanium and Silicon. Phys. Rev. 1954, 94, 42-49.

(45) Sze, S. M., Semiconductor Sensors; Wiley: New York 1994.

(46) Horcas, I.; Fernandez, R.; Gomez-Rodriguez, J. M.; Colchero, J.; Gomez-Herrero, J.; Baro, A. M. WSXM: A Software for Scanning Probe Microscopy and a Tool for Nanotechnology. Rev. Sci. Instrum. 2007, 78, 013705. 
Table of Contents Image:

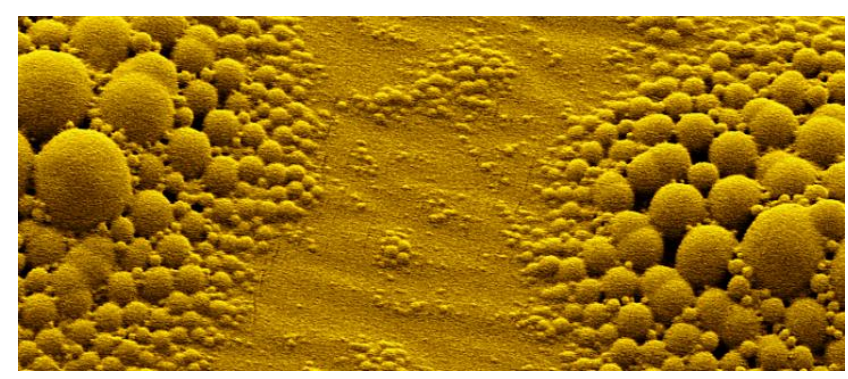

